\title{
In-medium omega meson mass and quark condensate in a Nambu Jona-Lasinio model constrained by recent experimental data
}

\author{
R. Huguet, J.C. Caillon and J. Labarsouque \\ Université Bordeaux 1; CNRS/IN2P3; \\ Centre d'Etudes Nucléaires de Bordeaux-Gradignan, UMR 5797 \\ Chemin du Solarium, BP120, 33175 Gradignan, France
}

\begin{abstract}
We have determined the relation between the in-medium $\omega$ meson mass and quark condensate in the framework of a Nambu Jona-Lasinio model constrained by some recent experimental data on the meson properties in nuclei. In addition to the usual four-quark interactions, we have included eight-quark terms in the Lagrangian. The parameters of this model have been determined using the meson properties in the vacuum as well as in the medium. More particularly, we have constrained both the in-medium pion decay constant to the value measured in experiments on deeply bound pionic atoms and the in-medium $\omega$ meson mass to the experimental value obtained either by the TAPS collaboration or by the E325 experiment at KEK. Our results are compared to several scaling laws and in particular to that of Brown and Rho.
\end{abstract}

PACS numbers: 12.39.Fe; 12.39.Ki; 14.40.-n; 21.65.+f

Keywords: Nambu-Jona-Lasinio model, in-medium $\omega$ meson mass, Brown and Rho scaling

\section{INTRODUCTION}

These last years, much attention has been focussed on the modification of hadron properties in nuclear environment and more particularly in the sector of the light vector mesons. The hope is that this modification could shed some light on prominent features of QCD at low energy. In particular, the knowledge of the dependence of the in-medium vector meson mass on the quark condensate is essential to a better understanding of the role played by the chiral structure of the QCD vacuum.

Experimentally, an indirect indication of the modification of hadron properties in the medium has been provided by the dilepton production measurements in relativistic heavy-ion collisions, like for example, experiments from CERES [1] and HELIOS [2] collaborations. However, the interpretation in terms of a reduction of the $\rho$ mass is still controversial. Recently, new experiments using proton-induced nuclear reactions [3], or $\gamma-A$ reactions [4] have provided a more clear experimental signature of the in-medium modifications of the $\omega$ mesons. In particular, the modification in nuclei of the $\omega$ meson has been investigated in photoproduction experiments by the TAPS collaboration[4] and its mass was found to be $m_{\omega}^{*}=722_{-4}^{+4}$ (stat) ${ }_{-5}^{+35}$ (syst) $\mathrm{MeV}$ at 0.6 times the saturation density of nuclear matter. The same order of magnitude, a $9 \%$ decrease of the in-medium $\omega$ mass at saturation, has been observed by Naruki et al. [3] in $12 \mathrm{GeV}$ proton-nucleus reactions (E325/KEK).

On the other hand, experimental indications of the in-medium modification of the quark condensate, $\langle\bar{q} q\rangle$, can be obtained, for example, in experiments on deeply bound pionic atoms. Indeed, by deducing the isovector $\pi N$ interaction parameter in the pion-nucleus potential from the binding energy and width of deeply bound 1s states of $\pi^{-}$in heavy nuclei, the in-medium pion decay constant, $f_{\pi}^{*}$, can be extracted [5, [6]. The quark condensate is then connected to $f_{\pi}^{*}$ through the Gell-Mann-Oaks-Renner relation. The observed enhancement of the isovector $\pi N$ interaction parameter over the free $\pi N$ value indicates a reduction of the pion decay constant in the medium which was found $[6]$ to be $f_{\pi}^{* 2} / f_{\pi}^{2}=0.64$ at saturation density of nuclear matter.

¿From a theoretical point of view, starting from the assumption of Harada and Yamawaki 7] on the "vector manifestation" of chiral symmetry in which a hidden local symmetry theory is matched to QCD, Brown and Rho proposed [8] that, up to the saturation density, the vector meson mass in medium, $m_{V}^{*}$, scales according to the approximative relation : $m_{V}^{*} / m_{V} \sim\left[\langle\bar{q} q\rangle /\langle\bar{q} q\rangle_{0}\right]^{1 / 2}$ (where $\langle\bar{q} q\rangle$ and $\langle\bar{q} q\rangle_{0}$ are respectively the in-medium and vacuum quark condensates). In quite different frameworks, like, for example, in finite density QCD sum rule calculations 9, 10, 11, 12, 13] or in the Nambu Jona-Lasinio model (NJL) 14], the relation between the in-medium vector meson mass and quark condensate is not so clear and thus more complicated to handle.

The recent experimental data, like those previously mentioned, should provide stringent tests for the models and for the relation between the in-medium $\omega$ meson mass and quark condensate. An indication on the consequences of these new constraints could be obtained by enforcing them in quark models incorporating the most prominent features of QCD. In this context, the NJL model[15] appears as a good candidate since it allows a dynamical description of both the breaking of chiral symmetry and of the modification of the in-medium $\omega$ meson mass.

In this work, we have determined the dependence of the in-medium $\omega$ meson mass on the quark condensate in a 
NJL model constrained by in-medium meson properties in accordance with recent experimental data. In addition to the usual four-quark interactions, we have included eight-quark terms in the NJL Lagrangian. The parameters of this model have been determined using the meson properties in the vacuum as well as in the medium through the pion decay constant and $\omega$ meson mass. More particularly, the in-medium pion decay constant has been constrained by the value obtained in an experiment on deeply bound pionic atoms $[6]$ and we have fixed the in-medium $\omega$ meson mass to the experimental values determined by the TAPS collaboration[4] or by the E325 experiment at KEK[3]. These inmedium changes of meson properties arise from dynamical chiral symmetry restoration at the quark mean-field-RPA level as well as from more complicated quark-gluon excitations usually parametrized in terms of many-body hadronic interactions. Considering the importance of the role played by the chiral structure of the QCD vacuum, concerning the $\omega$ meson mass and the pion decay constant, we made the assumption that, to leading order in nuclear density, the main contribution comes from dynamical chiral symmetry restoration at the quark mean-field-RPA level. Our results will be compared to several scaling laws and in particular to that of Brown and Rho.

\section{FORMALISM}

We consider the following chirally invariant two-flavor NJL Lagrangian 15] up to eight-quark interaction terms :

$$
\begin{aligned}
\mathcal{L}= & \bar{q}\left[i \gamma_{\mu} \partial^{\mu}-m_{0}\right] q+g_{1}\left[(\bar{q} q)^{2}+\left(\bar{q} i \gamma_{5} \tau q\right)^{2}\right]-g_{2}\left(\bar{q} \gamma_{\mu} q\right)^{2} \\
& +g_{3}\left[(\bar{q} q)^{2}+\left(\bar{q} i \gamma_{5} \tau q\right)^{2}\right]\left(\bar{q} \gamma_{\mu} q\right)^{2}+g_{4}\left[(\bar{q} q)^{2}+\left(\bar{q} i \gamma_{5} \tau q\right)^{2}\right]^{2},
\end{aligned}
$$

where $q$ denotes the quark field with two flavor $\left(N_{f}=2\right)$ and three color $\left(N_{c}=3\right)$ degrees of freedom and $m_{0}$ is the diagonal matrix of the current quark masses (here in the isospin symmetric case). The second and third terms of Eq11 represent local four-quark interactions while those proportional to $g_{3}$ and $g_{4}$ are eight-quark interactions. Let us recall that, in two-flavor models, the t'Hooft six fermion interaction term can be rewrited in terms of the four-quark interactions considered here[16]. We have not considered the term $\left(\bar{q} \gamma_{\mu} q\right)^{4}$ since, as in the nucleonic NJL model [17], it leads to a violation of the causality at high density.

The Dirac equation for a quark in mean-field approximation is given by :

$$
\left[i \gamma_{\mu} \partial^{\mu}-m_{0}-2 g_{2} \gamma_{0}\left\langle\bar{q} \gamma_{0} q\right\rangle+2 g_{1}\langle\bar{q} q\rangle+2 g_{3}\langle\bar{q} q\rangle\left\langle\bar{q} \gamma_{0} q\right\rangle^{2}+4 g_{4}\langle\bar{q} q\rangle^{3}\right] q=0
$$

which defines a dynamical constituent-quark mass :

$$
m=m_{0}-2 g_{1}\left(1+\frac{g_{3} N_{f}^{2} N_{c}^{2} \rho_{B}^{2}}{4 g_{1}}+\frac{2 g_{4}}{g_{1}}\langle\bar{q} q\rangle^{2}\right)\langle\bar{q} q\rangle,
$$

generated by a strong scalar interaction of the quark with the Dirac vacuum. In the gap equation (Eq 3 ), the quark condensate $\langle\bar{q} q\rangle$ can be written as :

$$
\langle\bar{q} q\rangle=-i \int \frac{d^{4} k}{(2 \pi)^{4}} \operatorname{Tr} S(k),
$$

where here Tr denotes traces over color, flavor and spin. In Eq廿 $S(k)$ represents the in-medium quark propagator defined as :

$$
S(k)=\frac{1}{\gamma_{\mu} k^{\mu}-m+i \varepsilon}+i \pi \frac{\gamma_{\mu} k^{\mu}+m}{E_{k}} \delta\left(k_{0}-E_{k}\right) \theta\left(k_{F}-|\mathbf{k}|\right),
$$

where $E_{k}=\sqrt{\mathbf{k}^{2}+m^{2}}$ and $k_{F}$ is the quark Fermi momentum. The baryonic density is related to the total quark density by $\rho_{B}=\frac{1}{3} \rho_{q}$. The quark condensate is divergent due to the loop integrals and requires an appropriate regularization procedure. As many authors [14, 18], we introduce a three-momentum cutoff $\Lambda$ which has the least impact on medium parts of the regularized integrals, in particular at zero temperature [18]. Thus, after the regularization procedure, the quark condensate is given at each density by : 


$$
\langle\bar{q} q\rangle=-\frac{N_{f} N_{c}}{\pi^{2}} \int_{k_{F}}^{\Lambda} \frac{m k^{2} d k}{E_{k}} .
$$

As usual, the $\omega$ meson mass is obtained by solving the Bethe-Salpeter equation in the quark-antiquark channel. First, we define the quark-antiquark polarization operator in the $\omega$ channel by :

$$
\begin{aligned}
\Pi_{\omega}^{\mu \nu}\left(q^{2}\right) & =-i \int \frac{d^{4} p}{(2 \pi)^{4}} \operatorname{Tr}\left[i \gamma^{\mu} i S(p+q / 2) i \gamma^{\nu} i S(p-q / 2)\right] \\
& =\left(-g^{\mu \nu}+\frac{q^{\mu} q^{v}}{q^{2}}\right) \Pi_{\omega}\left(q^{2}\right),
\end{aligned}
$$

where $S(k)$ is given by Eq 5 . Using the same regularization procedure as for the quark condensate, we obtain :

$$
\Pi_{\omega}\left(q^{2}\right)=\frac{N_{f} N_{c}}{12 \pi^{2}} q^{2} \int_{4\left(p_{F}^{2}+m^{2}\right)}^{4\left(\Lambda^{2}+m^{2}\right)} \frac{\sqrt{1-4 m^{2} / \kappa^{2}}}{q^{2}-\kappa^{2}}\left(1+\frac{2 m^{2}}{\kappa^{2}}\right) d \kappa^{2} .
$$

The in-medium $\omega$ meson mass, $m_{\omega}^{*}$, is then determined by the pole structure of the $T$-matrix, i.e. by the condition :

$$
1-2\left(g_{2}-g_{3}\langle\bar{q} q\rangle^{2}\right) \Pi_{\omega}\left(q^{2}=m_{\omega}^{* 2}\right)=0 .
$$

We also need the pion mass and decay constant to adjust the model parameters. In the pseudo-scalar channel, the polarization reads :

$$
\Pi_{\pi}\left(q^{2}\right)=\frac{\langle\bar{q} q\rangle}{m}+N_{c} N_{f} q^{2} I\left(q^{2}\right)
$$

with

$$
I\left(q^{2}\right)=\frac{1}{8 \pi^{2}} \int_{4\left(\Lambda^{2}+m^{2}\right)}^{4\left(p_{F}^{2}+m^{2}\right)} \frac{1}{q^{2}-\kappa^{2}} \sqrt{1-\frac{4 m^{2}}{\kappa^{2}}} d \kappa^{2} .
$$

The in-medium $\pi$ meson mass, $m_{\pi}^{*}$, and decay constant, $f_{\pi}^{*}$, are then given respectively by :

$$
\begin{gathered}
1-2\left(g_{1}+\frac{g_{3} N_{f}^{2} N_{c}^{2} \rho_{B}^{2}}{4}+2 g_{4}\langle\bar{q} q\rangle^{2}\right) \Pi_{\pi}\left(q^{2}=m_{\pi}^{* 2}\right)=0, \\
f_{\pi}^{*}=N_{c} N_{f} g_{\pi q q}^{*} m I\left(q^{2}=m_{\pi}^{* 2}\right),
\end{gathered}
$$

where the in-medium pion-quark-quark coupling constant, $g_{\pi q q}^{*}$, is obtained by :

$$
g_{\pi q q}^{* 2}=\left[\frac{d \Pi_{\pi}\left(q^{2}\right)}{d q^{2}}\right]_{q^{2}=m_{\pi}^{* 2}}^{-1}
$$

\section{RESULTS}

We have six free parameters : the cutoff $\Lambda$, the bare quark mass $m_{0}$, and the coupling constants $g_{1}, g_{2}, g_{3}$ and $g_{4}$. As usual, we have used for the fitting procedure the three following constraints : the pion mass $m_{\pi}=135 \mathrm{MeV}$, the pion decay constant $f_{\pi}=92.4 \mathrm{MeV}$ and the $\omega$ meson mass $m_{\omega}=782 \mathrm{MeV}$ in vacuum. Since additional constraints like, for example, the value of the quark condensate in vacuum, do not allow to determine the cutoff $\Lambda$ unambiguously, as often done [18], we have considered several values for $\Lambda$ or equivalently several values of the in-vacuum constituent 
quark mass, $m_{v a c}$, values ranging between $400 \mathrm{MeV}$ and $500 \mathrm{MeV}$. Such a rather large mass prevents the $\omega$ meson to be unstable against decay into a quark-antiquark pair since $m_{\omega}^{*}$ is always lower than $2 m$ for every density. Note that smaller constituent quark masses can be obtained in NJL models which take the confinement into account by including Polyakov loops [19] or using a confining interaction [20] in the Lagrangian. As already mentioned, in addition to these in-vacuum constraints, we have also chosen to take into account recent experimental results which provide constraints in the medium. In particular, we have fixed $f_{\pi}^{* 2}\left(\rho_{B}=\rho_{0}\right) / f_{\pi}^{2}=0.64$ (where $\rho_{0}$ is the saturation density of nuclear matter) in accordance with what is obtained in experiments on deeply bound pionic atoms [6]. Moreover, the in-medium $\omega$ meson mass has been constrained to reproduce the experimental central value obtained either by the TAPS collaboration [4],$m_{\omega}^{*}\left(\rho_{B}=0.6 \rho_{0}\right)=722 \mathrm{MeV}$, or by the E325 experiment at KEK $[3], m_{\omega}^{*}\left(\rho_{B}=\rho_{0}\right)=711$ $\mathrm{MeV}$. Thus, two families of parametrization sets denoted respectively by TAPS and KEK will be considered. Note that once $m_{\pi}, f_{\pi}, m_{\omega}, f_{\pi}^{*}, m_{\omega}^{*}$ and $m_{v a c}$ are fixed, all the parameters are determined unambiguously.

The results are shown on Fig.1 where we have plotted $m_{\omega}^{*} / m_{\omega}$ as a function of $\langle\bar{q} q\rangle /\langle\bar{q} q\rangle_{0}$ for the two parametrization sets TAPS and KEK. Note that $\langle\bar{q} q\rangle /\langle\bar{q} q\rangle_{0}=0.8$ corresponds to a baryonic density close to the saturation one. The shaded areas correspond to values of the $\omega$ meson mass for a constituent quark mass ranging from $400 \mathrm{MeV}$ to 500 $\mathrm{MeV}$. As we can see, these areas are rather narrow and the results are thus only weakly dependent on the value of $m_{\text {vac }}$ used.

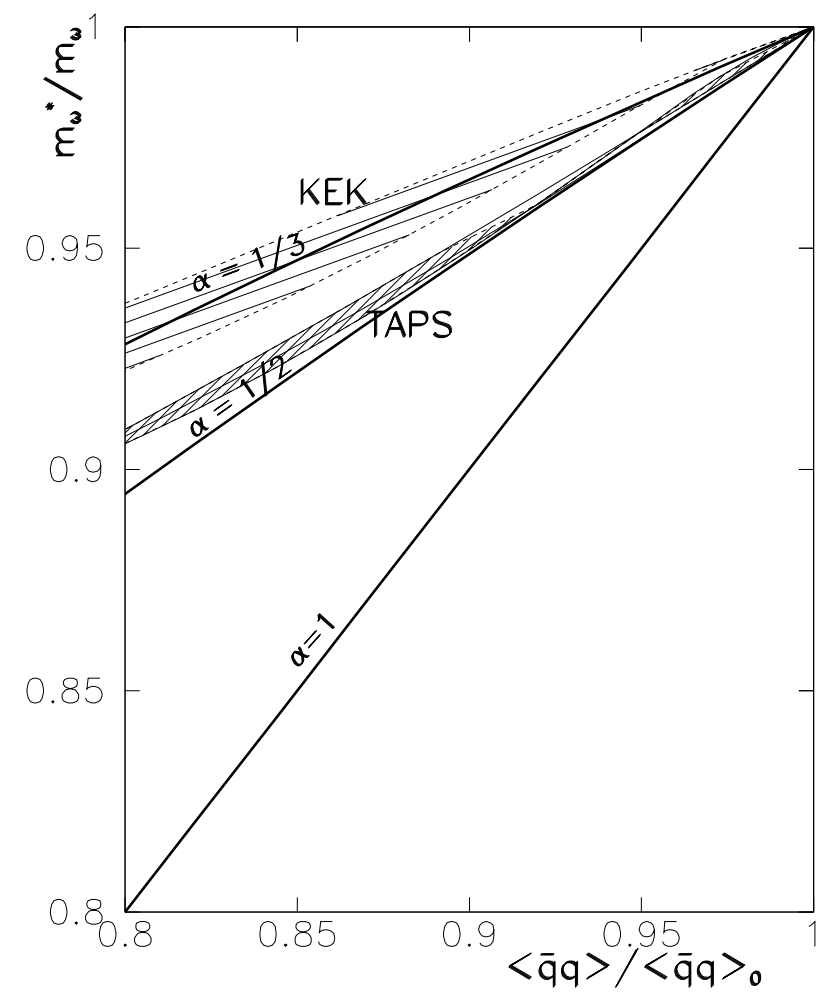

FIG. 1: In-medium $\omega$ meson mass as a function of the quark condensate. The shaded areas correspond to values obtained for a constituent quark mass in the range $400<m_{v a c}<500 \mathrm{MeV}$. The full lines represent the scaling laws given by Eq15 for $\alpha=1 / 3,1 / 2$ and 1 .

In order to determine an approximate form for the relation between the vector meson mass and the quark condensate, we have considered scaling laws of the general form :

$$
\frac{m_{\omega}^{*}}{m_{\omega}}=\left[\langle\bar{q} q\rangle /\langle\bar{q} q\rangle_{0}\right]^{\alpha}
$$

Any value of $\alpha$ can be considered but we have chosen to show here the results for $\alpha=1 / 2$ which corresponds to the Brown and Rho scaling and for neighboring values: $\alpha=1 / 3$ and $\alpha=1$. 
The full lines on Fig.1 represent the scaling laws given by Eq 15 for $\alpha=1 / 3,1 / 2$ and 1 . A rather good agreement with the case $\alpha=1 / 2$ corresponding to the Brown and Rho scaling law is obtained for the TAPS parametrization set while the KEK result clearly favours $\alpha=1 / 3$. Assuming Eq15 for the scaling law, this result can be understood since to leading order $\left(\langle\bar{q} q\rangle /\langle\bar{q} q\rangle_{0}\right)^{\alpha} \simeq 1-\alpha\left(1-\langle\bar{q} q\rangle /\langle\bar{q} q\rangle_{0}\right)$ and $\langle\bar{q} q\rangle /\langle\bar{q} q\rangle_{0} \simeq 1-\beta \rho / \rho_{0}$ with $\beta=\sigma_{N} \rho_{0} / f_{\pi}^{2} m_{\pi}^{2}$ where $\sigma_{N}$ is the $\pi N$ sigma term. The TAPS or KEK results can then be used to determine the product $\alpha \beta$ obtained by eliminating the quark condensate in $m_{\omega}^{*} / m_{\omega}$. Using the value $\sigma_{N} \simeq 35 \mathrm{MeV}$ obtained in the NJL model for $m \simeq 450$ $\mathrm{MeV}$, the TAPS and KEK results provide respectively $\alpha$ close to $1 / 2$ and $1 / 3$ in agreement with our full calculation.

On the other hand, the experimental values of the in-medium $\omega$ meson mass are not determined unambiguously. In particular, the TAPS collaboration obtained a rather large uncertainty including statistical and systematical errors. By taking into account such an uncertainty in our calculation, we have found that the scaling law (Eq 15) with $\alpha=1$ is clearly ruled out but the case $\alpha=1 / 3$ is not totally excluded. However, note that the central value of the TAPS result clearly favours $\alpha=1 / 2$.

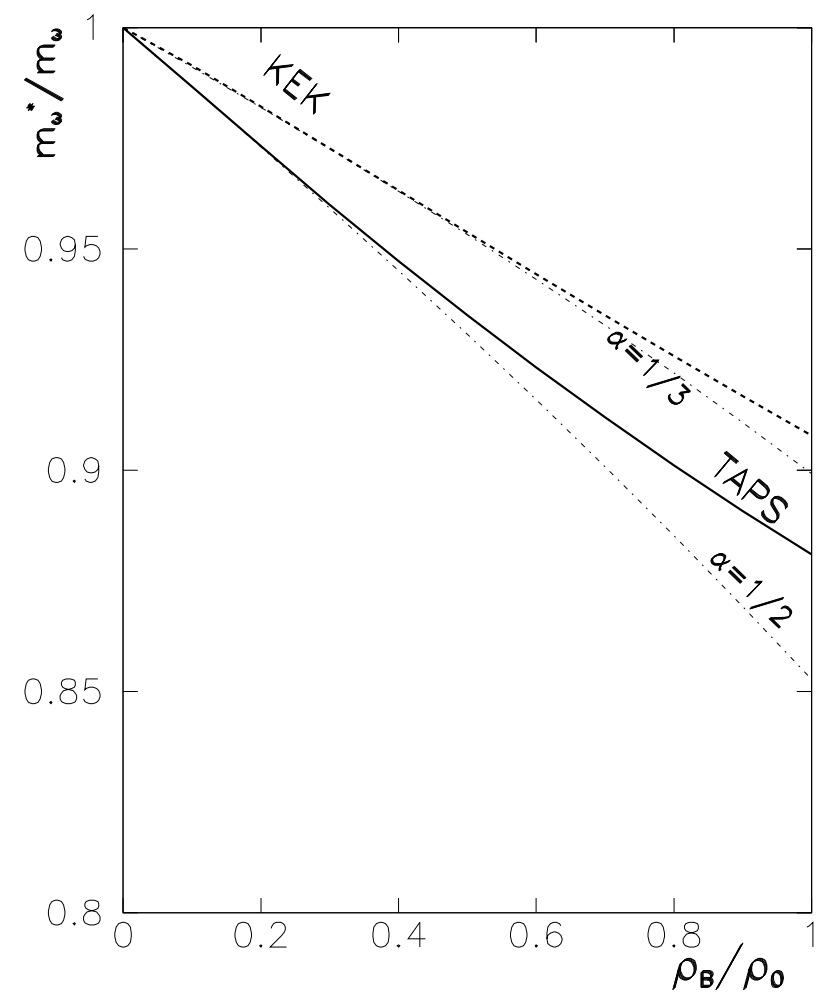

FIG. 2: In-medium $\omega$ meson mass as a function of the baryonic density for the TAPS (solid curve) and KEK (dashed curve) parametrization sets. For comparison the scaling laws (15) with $\alpha=1 / 2$ and $1 / 3$ are also shown (dot-dashed curves).

Let us now determine how the quark condensate and the $\omega$ meson mass depend on the baryonic density for central values of the constituent quark mass, i.e. for $m_{v a c}=450 \mathrm{MeV}$, respectively for the TAPS and KEK parametrizations. The parameters of the NJL model are then $\Lambda=575 \mathrm{MeV}, m_{0}=5.6 \mathrm{MeV}, g_{1} \Lambda^{2}=2.53, g_{2} \Lambda^{2}=5.20, g_{3} \Lambda^{8}=62.5$ and $g_{4} \Lambda^{8}=2.27$ for the TAPS parametrization and $\Lambda=575 \mathrm{MeV}, m_{0}=5.6 \mathrm{MeV}, g_{1} \Lambda^{2}=2.58, g_{2} \Lambda^{2}=4.12$, $g_{3} \Lambda^{8}=12.4$ and $g_{4} \Lambda^{8}=1.22$ for the KEK one. Let us mention that, whatever the parametrization considered, the model provides a quark condensate in the vacuum $\langle\bar{u} u\rangle^{1 / 3}=-240 \mathrm{MeV}$ in good agreement with the lattice calculations: $\langle\bar{u} u\rangle^{1 / 3}=-(231 \pm 4 \pm 8 \pm 6) \mathrm{MeV}$ [21] and a critical density close to four times the saturation one. On Fig.2, we have plotted $m_{\omega}^{*} / m_{\omega}$ (solid curves) for the two parametrizations TAPS and KEK as well as $\left[\langle\bar{q} q\rangle /\langle\bar{q} q\rangle_{0}\right]^{\alpha}$ for $\alpha=1 / 2$ (dashed curve) and $\alpha=1 / 3$ (dot-dashed curve), as a function of the dimensionless baryonic density $\rho_{B} / \rho_{0}$. As we can see, the density dependences of the in-medium $\omega$ meson mass obtained with the TAPS and KEK parametrizations lead to a drop close to $10 \%$ at saturation density ( $9 \%$ for KEK and $12 \%$ for TAPS). As already 
discussed, to a good level of approximation, the in-medium $\omega$ meson mass determined using the KEK result varies as a function of the baryonic density like the third root of the quark condensate. On the other hand, using the TAPS result, the density dependence of the in-medium $\omega$ meson mass is not very much different from the square root of the quark condensate reflecting the fact that the results follow approximately the Brown and Rho scaling law up to the saturation density.

\section{CONCLUSION}

We have determined the in-medium $\omega$ meson mass and quark condensate in a NJL model with eight quark interaction terms. The parameters of this model have been determined using the meson properties in the vacuum but also in the medium through the value of the pion decay constant obtained in experiments on deeply bound pionic atoms as well as the $\omega$ meson mass measured either by the TAPS collaboration or by the E325/KEK experiment. When the in-medium $\omega$ meson mass is constrained to the experimental data obtained by the TAPS collaboration, the Brown and Rho scaling law is approximately recovered. On the other hand, when the KEK result is used, the in-medium $\omega$ meson mass varies rather like the third root of the quark condensate. However, in both cases, this corresponds to a drop of the $\omega$ meson mass at saturation density close to $10 \%$, a result which is lower than those found in QCD sum rule calculations where a decrease close to $15-25 \%$ is generally obtained 9,12 , 13].

[1] P. Wurm for the CERES Collaboration, Nucl. Phys. A590, 103c (1995).

[2] M. Masera for the HELIOS Collaboration, Nucl. Phys. A590, 93c (1995).

[3] M. Naruki et al., Phys. Rev. Lett. 96, 092301 (2006).

[4] D. Trnka et al., Phys. Rev. Lett. 94, 192303 (2005).

[5] H. Geissel et al., Phys. Lett. B549, 64 (2002).

[6] K. Suzuki et al. Phys. Rev. Lett. 92, 072302 (2004).

[7] M. Harada and K. Yamawaki, Phys. Rev. Lett. 86, 757 (2001); Phys. Rep. 381, 1 (2003).

[8] G. E. Brown and M. Rho, Phys. Rep. 398, 301 (2004).

[9] T. Hatsuda and S. H. Lee, Phys. Rev. C46, R34 (1992); T. Hatsuda, S. H. Lee and H. Shiomi, Phys. Rev. C52, 3364 (1995).

[10] M. Asakawa and C. M. Ko, Phys. Rev. C48, R526 (1993).

[11] Y. Koike, Phys. Rev. C51, 1488 (1995).

[12] X. Jin, D. B. Leinweber, Phys. Rev. C52, 3344 (1995).

[13] F. Klingl, N. Kaiser and W. Weise, Nucl. Phys.A 624, 527 (1997).

[14] V. Bernard and U. G. Meissner, Nucl. Phys. A489, 647 (1988).

[15] Y. Nambu and G. Jona-Lasinio, Phys. Rev. 122, 345 (1961); Phys. Rev. 124, 246 (1961).

[16] S. P. Klevansky Rev. Mod. Phys. 64, 649 (1992).

[17] I.N. Mishustin, L.M. Satarov and W. Greiner Phys. Rep. 391, 363 (2004).

[18] M. Buballa, Phys. Rep. 407, 205 (2005).

[19] C. Ratti, M.A. Thaler and W. Weise Phys. Rev. D73, 14019 (2006).

[20] L.S. Celenza, B. Huang and C.M. Shakin Phys. Rev. C59, 1030 (1999).

[21] L. Giusti, F. Rapuano, M. Talevi and A. Vladikas, Nucl. Phys. B 538, 249 (1999). 